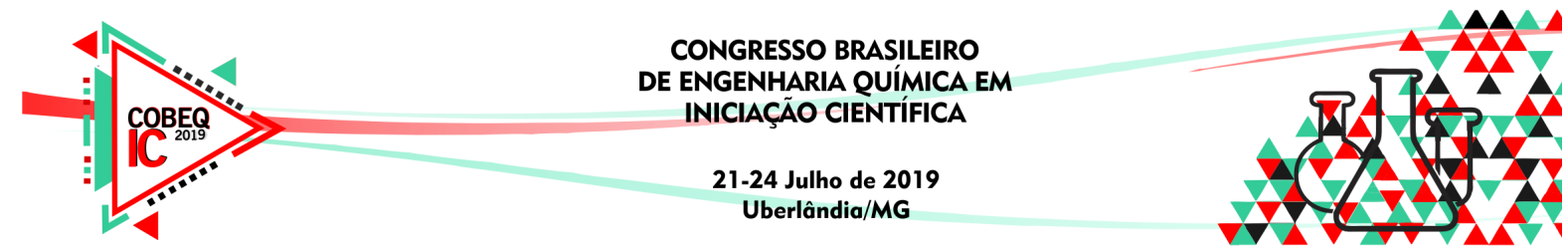

\title{
AVALIAÇÃO DOS EFEITOS DAS VARIÁVEIS DE PROJETO E OPERACIONAL NO DESEMPENHO DE UM HIDROCICLONE CONCENTRADOR
}

\author{
A. R. IGARASHI ${ }^{1}$, G. L. D. PEREIRA ${ }^{1}$, F. N. C. ALMEIDA ${ }^{1}$, D. C. S. MAIA ${ }^{1}$ e N. C. \\ PEREIRA $^{1}$ \\ ${ }^{1}$ Universidade Estadual de Maringá, Departamento de Engenharia Química \\ E-mail para contato: ra102612@uem.br
}

\begin{abstract}
RESUMO - Hidrociclones são equipamentos que podem ser empregados com o intuito de classificar partículas ou de concentrar uma das correntes que saem do equipamento. A eficiência de separação de partículas e a recuperação de fluido pode ser melhorada modificando os parâmetros geométricos ou o fluxo característico. Desta forma, o presente trabalho tem como objetivo identificar os parâmetros geométricos de um hidrociclone que mais influenciam na recuperação de fluido através do seu overflow. Os hidrociclone foram projetados de acordo com um planejamento fatorial fracionário $2_{I I I}^{3-1}$ e simulados via CFD. No estudo foram avaliados a influência do diâmetro do overflow ou diâmetro do vortex finder $\left(\mathrm{D}_{\mathrm{o}}\right)$, o diâmetro do underflow $\left(\mathrm{D}_{\mathrm{u}}\right)$, a altura da região cilíndrica do hidrociclone $\left(\mathrm{H}_{\mathrm{c}}\right)$, a altura do vortex finder $\left(\mathrm{H}_{\mathrm{vf}}\right)$, a vazão de alimentação do hidrociclone $\left(\mathrm{Q}_{\mathrm{f}}\right)$ e o ângulo de abertura da seção cônica $(\theta)$. Dos seis parâmetros apresentados, os fatores que mais influenciaram significativamente na recuperação de fluido foram $\mathrm{D}_{\mathrm{u}}$ seguido de $\mathrm{D}_{\mathrm{o}}$. O diâmetro do underflow influenciou negativamente e o diâmetro do overflow influenciou positivamente na recuperação de solução.
\end{abstract}

\section{INTRODUÇÃO}

Hidrociclones fazem parte de um grupo de dispositivos de classificação de sólidos e líquidos, compostos por uma construção simples, ausente de qualquer peça móvel. Tais estruturas realizam a separação de matéria dispersa em uma corrente de fluido, sendo amplamente utilizados por permitirem a obtenção de uma alta eficiência de separação, além de fornecerem vantagens, como estrutura simples, baixo custo e consumo de energia, grande capacidade de carga em pequeno volume, além de necessitarem de pouca manutenção e módulos de suporte Sripriya et al. (2007).

Em hidrociclones convencionais, compostos por uma estrutura cilíndrica e cônica, como apresentado na Figura 1, observa-se a presença de duas saídas, cada uma posicionada em um mesmo eixo, mas opostas uma a outra. A primeira se encontra na porção superior do ciclone, sendo caracterizada por um tubo interno, o vortex finder, que conecta o interior do cilindro com a porção externa, esta saída é também denominada overflow. Enquanto a segunda saída se encontra na porção inferior da estrutura, denominada também como underflow. 


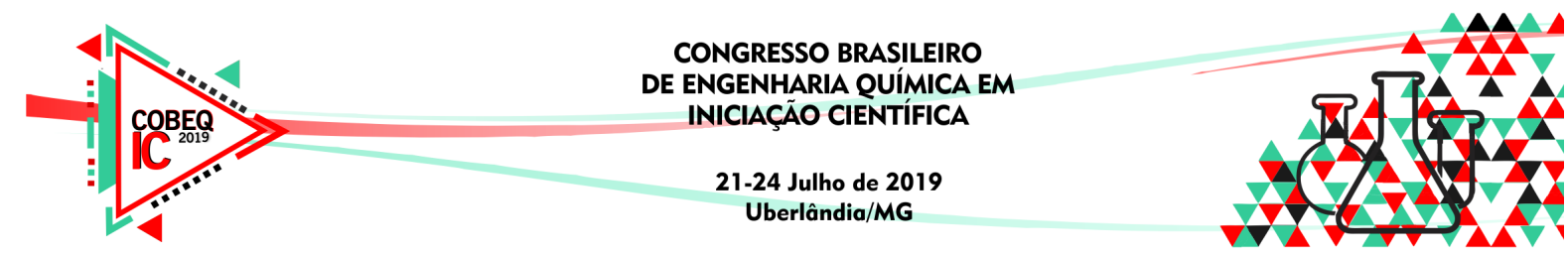

Figura 1 - Representação da estrutura de um hidrociclone.

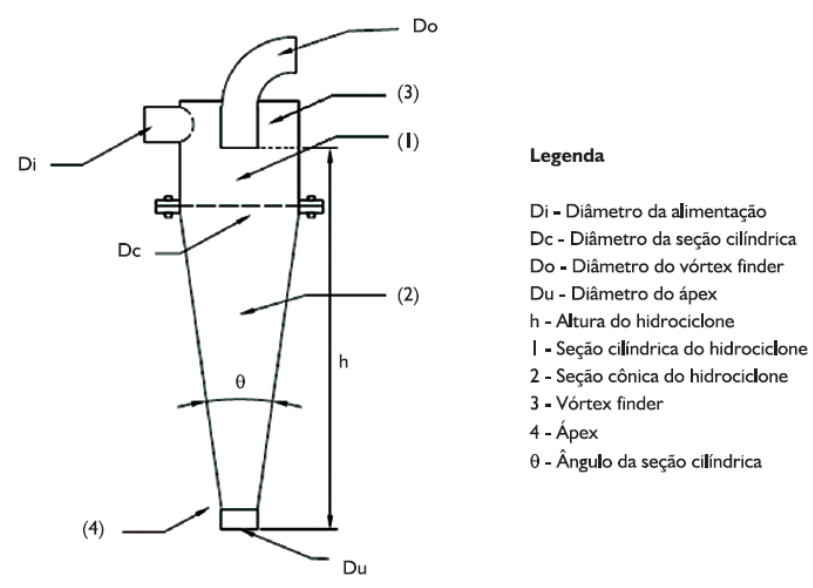

Fonte: Silva et al. (2012).

Em seu funcionamento, o equipamento converte o movimento linear proveniente do fluido quando acaba de passar pela entrada do hidrociclone, em um movimento angular que varia continuamente, desse modo, ocorre a exposiçãodas partículas dispersas no líquido à uma aceleração centrífuga, melhorando a taxa de sedimentação dos sólidos que se organizam dentro da estrutura do ciclone de acordo com sua diferença de tamanho, densidade e formato.

Os hidrociclones podem ser empregados com o intuito de classificar partículas ou de concentrar uma das correntes que saem do equipamento. Para um hidrociclone com relações geométricas específicas $\left(\mathrm{D}_{\mathrm{u}} / \mathrm{D}_{\mathrm{c}}, \mathrm{D}_{\mathrm{o}} / \mathrm{D}_{\mathrm{c}}, \mathrm{H}_{\mathrm{v}} / \mathrm{D}_{\mathrm{c}}, \mathrm{H}_{\mathrm{c}} / \mathrm{D}_{\mathrm{c}}\right.$ e $\left.\theta\right)$, mudanças aleatórias destes parâmetros podem melhorar, manter ou piorar o desempenho do dispositivo base (Kyriakidis et al., 2018). Por este motivo, o estudo destes acessórios torna-se essencial e necessário para a caracterização adequada de qualquer novo hidrociclone. Desta forma, o presente trabalho tem como objetivo identificar a influência dos parâmetros geométricos diâmetro inferior $\left(\mathrm{D}_{\mathrm{u}}\right)$, diâmetro superior $\left(\mathrm{D}_{\mathrm{o}}\right)$, altura do vortex finder $\left(\mathrm{H}_{\mathrm{vf}}\right)$, altura da seção cilíndrica $\left(\mathrm{H}_{\mathrm{c}}\right)$, vazão de entrada $\left(\mathrm{Q}_{\mathrm{f}}\right)$ e o ângulo de abertura $(\theta)$ de um hidrociclone na recuperação de fluido através do seu overflow. Os projetos de hidrociclones foram construídos baseados em um planejamento fatorial fracionário, e simulados via CFD.

\section{MATERIAIS E MÉTODOS}

\subsection{Sistema de partículas e solução}

O material particulado utilizado para a simulação foi o enxofre elementar, com densidade $1,960 \mathrm{~g} / \mathrm{cm}^{3}$ (Maia, 2015) e diâmetro médio de 1,093 $\pm 0,004 \mu \mathrm{m}$ (método DLS). A solução utilizada foi de FeEDTA, com densidade igual a $1,156 \mathrm{~g} / \mathrm{cm}^{3}$ (Maia, 2015). A viscosidade da suspensão foi considerada igual a $1,0 \mathrm{cP}$, pelo fato da concentração de material partícula ser de $0,3 \%$ em base mássica.

\subsection{Planejamento experimental}

Com o objetivo de identificar quais os fatores que tem influência significativa na recuperação de fluido, os hidrociclones foram projetados de acordo com um planejamento 


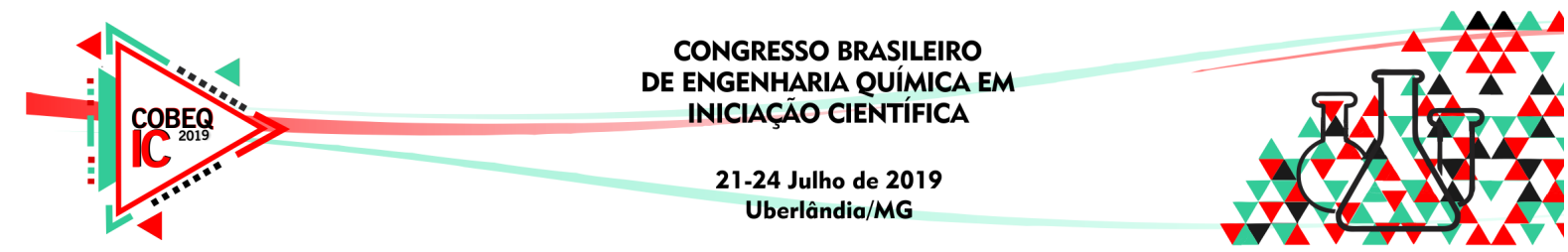

fatorial fracionado $2_{I I I}^{3-1}$, com resolução do tipo III. Nesse tipo de resolução, os efeitos principais não são estão relacionados com nenhum outro efeito principal, assumindo-se que as interações de ordem 2 ou superiores são desprezíveis com base no princípio da dispersão de efeitos.

Foram projetados 8 hidrociclones, variando os seguintes fatores: diâmetro do overflow $(1,32-3,95 \mathrm{~mm})$, diâmetro do underflow $(0,99-2,96 \mathrm{~mm})$, a altura da parte cilíndrica $(14,02$ - 42,06 mm), a altura do vortex finder $(2,17$ - 6,51), vazão de alimentação $(244-732$ $\mathrm{mL} / \mathrm{min})$ e ângulo do cone $(4,5$ - 13,5). Os valores dos níveis, baixo (-1) e alto (+1), foram obtidos variando em $\pm 50 \%$ a partir do dimensionamento da geometria pertencente à família Bradley, determinado em estudos preliminares. O software Design-Expert (V. 7.0.0) foi utilizado para a implementação e avaliação do design $2_{I I I}^{3-1}$. As médias foram comparadas por ANOVA-fatorial. O nível de significância foi estabelecido em $\alpha=0,05$.

\subsection{Simulação e recuperação de solução}

Para avaliar a recuperação de solução, os hidrociclones foram construídos e simulados no software ANSYS (versão 19.2, licença estudantil). Para a construção do modelo geométrico foi utilizado o pacote ANSYS SpaceClaim. As malhas cartesianas foram geradas no pacote ANSYS Meshing, empregando uma abordagem Euleriana-Lagrangiana. Para a resolução das equações diferenciais foi usado o Solver do pacote ANSYS Fluent, definindo a água como a fase contínua e partículas de enxofre como a fase discreta. O sistema foi resolvido usando o modelo de estresse Reynolds com um solver baseado em pressão e o método semi-implícito para pressão-ligado, algoritmo de equações (SIMPLES) para o par pressão-velocidade Vega-Garcia et al. (2018).

O gradiente usado na resolução foi o baseado nos últimos quadrados, juntamente com as equações de segunda ordem de pressão e momento linear. Neste sistema de equações também se utilizou as equações do modelo viscoso k-omega configuradas para o método power rule.

O desempenho dos hidrociclones na simulação foi avaliado com base na recuperação de solução, que é dado como a razão existente entre a vazão de suspensão que sai no overflow $\left(Q_{o}\right)$ e a vazão alimentada $\left(Q_{f}\right)$ no sistema (Equação 1). Quando o objetivo do hidrociclone é o espessamento, ou seja, a separação do líquido da fase sólida a variável resposta recuperação de solução deve ser avaliada.

$$
R=\frac{Q_{o}}{Q_{f}}
$$

\section{RESULTADOS E DISCUSSÃO}

Os dados obtidos por meio das simulações para a realização do planejamento fatorial para a recuperação de solução estão expostos na Tabela 1. A recuperação de solução no overflow variou de $11,08 \%$ a $99,00 \%$. Este grande intervalo entre as respostas estudadas indica que os fatores estudados e seus respectivos níveis foram escolhidos com sucesso.

Tabela 1 - Valores obtidos para o planejamento fatorial. 

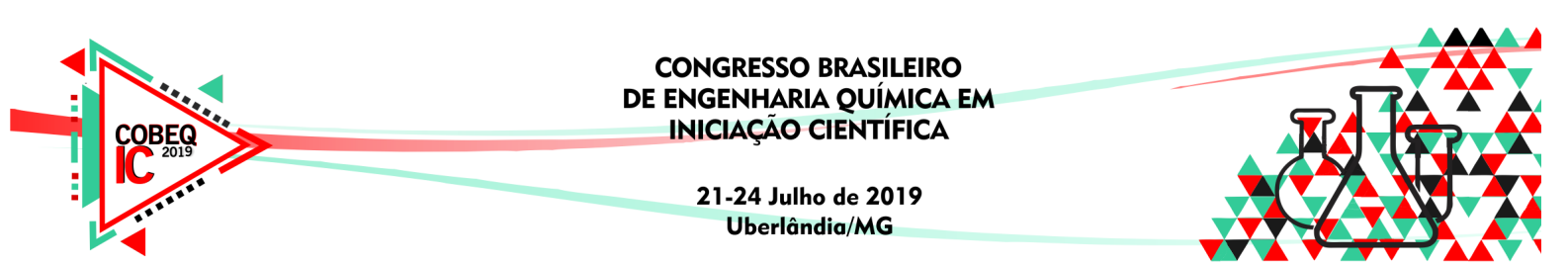

\begin{tabular}{|c|c|c|c|c|c|c|c|}
\hline Projeto & $\begin{array}{c}\mathbf{D}_{\mathbf{u}} \\
(\mathbf{m m})\end{array}$ & $\begin{array}{c}\mathbf{D}_{\mathbf{o}} \\
(\mathbf{m m})\end{array}$ & $\begin{array}{c}\mathbf{Q}_{\mathbf{f}} \\
(\mathbf{m L} / \mathbf{m i n})\end{array}$ & $\begin{array}{c}\boldsymbol{\theta} \\
\mathbf{(})\end{array}$ & $\begin{array}{c}\mathbf{H}_{\mathbf{v f}} \\
(\mathbf{m m})\end{array}$ & $\begin{array}{c}\mathbf{H}_{\mathbf{c}} \\
(\mathbf{m m})\end{array}$ & $\begin{array}{c}\text { Recuperação } \\
\mathbf{( \% )}\end{array}$ \\
\hline 1 & 0.985 & 1.315 & 244 & 13.5 & 6.51 & 42.06 & 92,15 \\
\hline 2 & 2.955 & 1.315 & 244 & 4.5 & 2.17 & 42.06 & 12,46 \\
\hline 3 & 0.985 & 3.945 & 244 & 4.5 & 6.51 & 14.02 & 99,00 \\
\hline 4 & 2.955 & 3.945 & 244 & 13.5 & 2.17 & 14.02 & 47,96 \\
\hline 5 & 0.985 & 1.315 & 732 & 13.5 & 2.17 & 14.02 & 58,27 \\
\hline 6 & 2.955 & 1.315 & 732 & 4.5 & 6.51 & 14.02 & 11,08 \\
\hline 7 & 0.985 & 3.945 & 732 & 4.5 & 2.17 & 42.06 & 97,26 \\
\hline 8 & 2.955 & 3.945 & 732 & 13.5 & 6.51 & 42.06 & 46,81 \\
\hline
\end{tabular}

A Figura 2 ilustra a contribuição dos efeitos principais na variável resposta. Os efeitos que se encontram acima do limite de Bonferroni, para um nível de significância de 5\%, são, certamente, significativos e os que se encontram abaixo do limite do t-valor certamente são não significativos. Desta forma, as variáveis de projeto $D_{u}$ e $D_{o}$ mostraram-se as que mais influenciaram no processo de recuperação de solução do hidrociclone.

Figura 2 - Efeitos estimados de parâmetros na recuperação de fluido de um hidrociclone, usando um delineamento fracionado $2_{I I I}^{3-1}$.

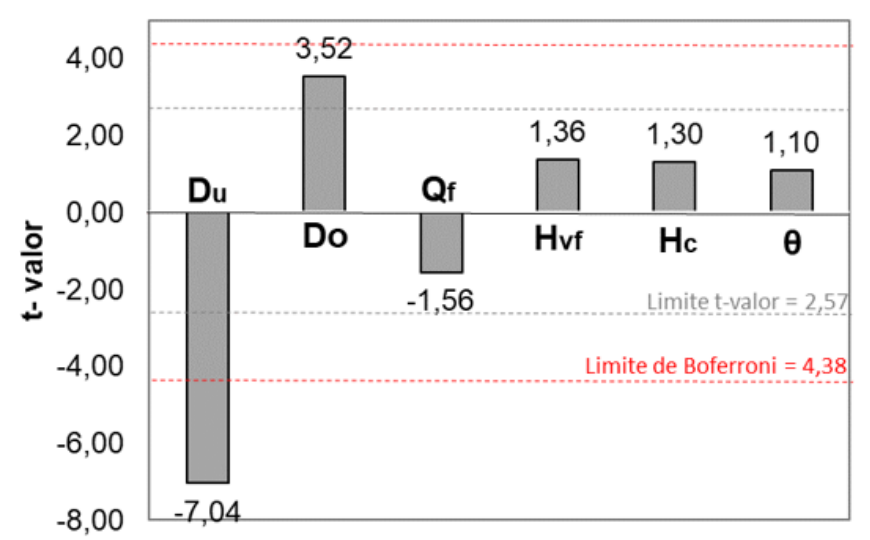

Observa-se na Figura 2, que o diâmetro do underflow $\left(\mathrm{D}_{u}\right)$ mostrou-se ser a variável que mais pesou negativamente na recuperação. O que está em conformidade com Kyriakidis et al. (2018), que divulgam que a diminuição no valor do diâmetro de saída inferior aumenta a restrição de fluxo para a saída inferior, ao mesmo tempo em que aumenta a velocidade axial de fluído para cima, sendo uma maior quantidade de fluido descarregada pela saída superior.

O diâmetro do overflow $\left(\mathrm{D}_{\mathrm{o}}\right)$ foi o fator que mais afetou positivamente a recuperação, estando de acordo com o proposto por Ahmed et al. (2007), segundo os quais o aumento no diâmetro do vortex finder aumenta o diâmetro da coluna de ar, o que resulta num acréscimo do volume de fluido destinado à saída superior.

Apesar das demais variáveis não terem apresentado efeito significativo na variável resposta (recuperação), o efeito de suas variáveis apresenta-se de acordo com a literatura. A vazão de entrada $\left(\mathrm{Q}_{\mathrm{v}}\right)$ relaciona-se diretamente com a recuperação. Segundo Jiang et al. (2018), tem-se que, com o aumento da vazão de fluido na entrada, há o consequente aumento na velocidade axial do vórtice interno no ciclone, o que resulta em uma maior vazão de fluido sendo descarregada através da saída superior da estrutura, levando a uma maior recuperação de solução. 


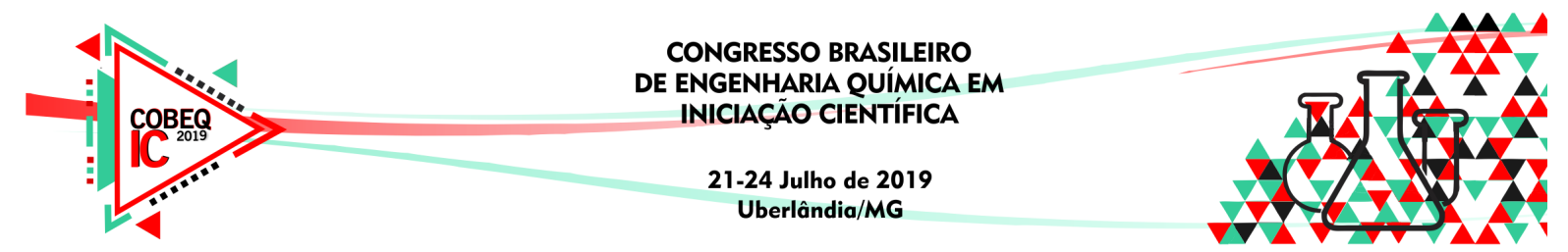

O ângulo de abertura do cone $(\theta)$ interfere positivamente na recuperação, o que está de acordo com o que fora proposto por Wang et al. (2006), segundo os quais, um aumento no valor do ângulo, acarreta em um volume de diminuto se encaminhando para a saída inferior e uma menor razão de fluxo de fluido. Tal efeito é observado pois há a um crescimento do diâmetro da coluna de ar no interior do ciclone.

A altura do vortex finder $\left(\mathrm{H}_{\mathrm{vf}}\right)$ favorece a recuperação de fluido, assim como relatado por Tang et al. (2015), no qual, segundo estes, com o crescimento no valor da altura do vortex finder a velocidade axial se dirigindo em direção à saída superior aumentava, resultando em uma maior quantidade de fluido seguindo este movimento forçado.

A altura da seção cilíndrica $\left(\mathrm{H}_{\mathrm{c}}\right)$ interfere de forma direta na recuperação de solução, assim como proposto por Wang et al. (2006), onde fora afirmado que, com o acréscimo da altura da seção cilíndrica tem-se uma diminuição na queda de pressão, fazendo com que haja uma menor razão entre a quantidade de fluido liberada pela saída inferior com a descarregada pela saída superior.

Após cada resposta ser analisada individualmente, um modelo linear simples foi proposto, empregando apenas as variáveis significativas $\left(\mathrm{D}_{\mathrm{u}}\right.$ e $\left.\mathrm{D}_{\mathrm{o}}\right)$. A análise de variância (ANOVA) foi utilizada para avaliar a adequação do modelo, apresentando $\mathrm{R}^{2}$ igual a $92,60 \%$ e p-valor $(0,0015)$ menor que 0,05 , indicando que o modelo é significativo. $O$ gráfico da superfície de resposta (Figura 3) foi desenhado variando as duas variáveis nos limites das condições estudadas, mantendo as demais constantes.

Figura 3 - Perfil de superfície para recuperação de fluido no overflow em função do diâmetro do underflow e overflow

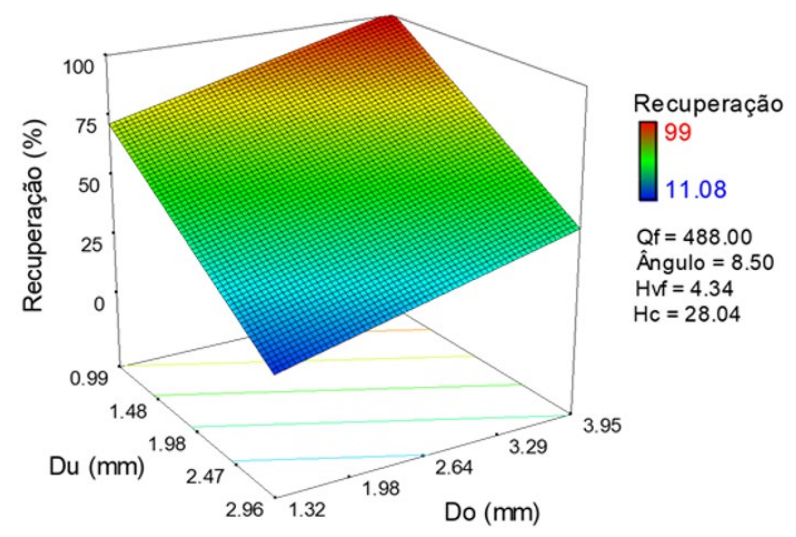

A Figura 3 ilustra os efeitos de $D_{u}$ e $D_{o}$ na recuperação de solução no hidrociclone. $O$ gráfico mostra que em baixo nível de $\mathrm{D}_{\mathrm{u}}$ e em alto nível de $\mathrm{D}_{\mathrm{o}}$ é possível obter a máxima recuperação de solução (100\%). No entanto, tecnicamente esta condição não é favorável para o processo de separação empregando um hidrociclone. Uma vez que, quando se tem uma recuperação de $100 \%$, significa que toda a solução que entrou, saiu pelo overflow, impedindo que ocorra uma separação do sólido-fluido. Sendo assim, é necessária uma avaliação da variável resposta eficiência de separação em conjunto com a variável recuperação, para que seja possível determinar uma região ótima para o dimensionamento do hidrociclone. 


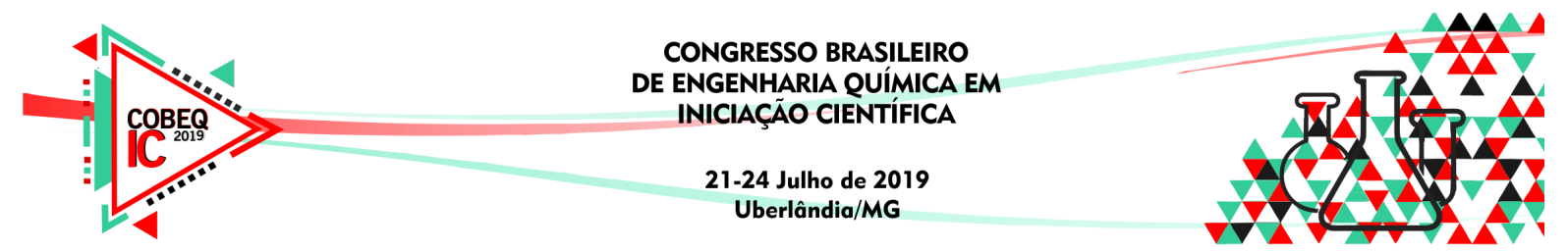

\section{CONCLUSÃO}

De acordo com os resultados obtidos, teve-se que fatores que mais afetaram a variável resposta estudada foram $D_{u}$, que influenciou na recuperação de forma negativa, e $D_{o}$ que a impactou de forma positiva. Enquanto que as demais variáveis, como $\mathrm{H}_{\mathrm{fv}}$, embora tenham influenciado na recuperação, fizeram de forma bem menos acentuada. O hidrociclone 3 fora aquele que apresentou o melhor valor de recuperação. Sugere-se em trabalhos futuros otimizar o dimensionamento do hidrociclone avaliando as variáveis repostas recuperação de solução e eficiência de separação simultaneamente.

\section{REFERÊNCIA}

AHMED, M. M.; IBRAHIM, G. A.; FARGHALY, M. G. Performance of three-product hydrocyclone Part II: Distribution of water recovery in the three product hydrocyclone. Journal of Eng. Sci., Vol 35, p. 815-828, 2007.

JIANG, J.; YING, R.; FENG, J.; WANG, W. Computational and Experimental Study of the Effect of Operating Parameters on Classification Performance of Compound Hydrocyclone. Math. Probl. in Eng., p.1-16, 2018.

KYRIADIKIS, Y. N.; SILVA, D. O.; BARROZO, M. A. S.; VIEIRA, L. G. M. Effect of variables related to the separation performance of a hydrocyclone with unprecedented geometric relationships. Powd. Technol., Vol 338, p. 645-653, 2018.

MAIA, D. C. S. Desenvolvimento de um processo de purificação de biogás para uso energético. Tese, PEQ, Universidade Estadual de Maringá, Brasil, 2015.

SILVA, A. C.; SILVA, E. S; MATOS, J. Modelo Empírico para Cálculo do Diâmetro de Corte Corrigido de Hidrociclones. Tecnol. Metal. Mater. Miner., Vol. 9, p. 302-308, 2012.

SRIPRIYA, R.; KAULASKAR, M. D.; CHAKRABORTY, S.; MEIKAP, B. C. Studies on the performance of a hydrocyclone and modeling for flow characterization in presence and absence of air core. Chem. Eng. Sci., Vol 62, p. 6391-6402, 2007.

TANG, B.; XU, Y.; SONG, X.; SUN, Z.; YU, J. Numerical study on the relationship between high sharpness and configurations of the vortex finder of a hydrocyclone by central composite design. Chem. Eng. Journal., Vol 278, p. 504-516, 2015.

VEGA-GARCIA, D.; BRITO-PARADA, P. R.; CILlIERS, J. J. Optimising small hydrocyclone design using 3D printing and CFD simulations. Chem. Eng. Journal., Vol 350 , p. 653-659, 2018.

WANG, B.; YU, A.B. Numerial study of particle-fluid flow in hydrocyclones with different body dimensions. Mine. Eng., Vol 19, p. 1022-1033, 2006. 